\title{
On the multiwavelength properties of several $\gamma$-ray detected narrow-line Seyfert 1 galaxies
}

\author{
Hui Yang ${ }^{* 1,2}$, Weimin Yuan ${ }^{1,2}$, Su Yao ${ }^{3,4}$, Hai-Wu Pan ${ }^{1}$ and S. Komossa ${ }^{5}$ \\ ${ }^{1}$ Key Laboratory of Space Astronomy and Technology, National Astronomical Observatories, \\ Chinese Academy of Sciences, Beijing 100012, China \\ ${ }^{2}$ School of Astronomy and Space Science, University of Chinese Academy of Sciences, $19 \mathrm{~A}$ \\ Yuquan Road, Beijing 100049, China \\ ${ }^{3}$ Kavli Institute for Astronomy and Astrophysics, Peking University, Beijing 100871, China \\ ${ }^{4}$ National Astronomical Observatories, Chinese Academy of Sciences, Beijing 100012, China \\ ${ }^{5}$ Max-Planck-Institut für Radioastronomie, Auf dem Hügel 69, D-53121 Bonn, Germany \\ E-mail: huiyangdnao.cas.cn, wmydnao.cas.cn
}

\begin{abstract}
The $\gamma$-ray detection from several radio-loud (RL) narrow-line Seyfert 1 (NLS1) galaxies has enabled us to study powerful relativistic jets in active galactic nuclei (AGNs) with smaller black hole masses and higher accretion rates than classical blazars. However, the sample of those $\gamma$ ray detected NLS1s available is still not large enough for a comprehensive and statistical study. We provide a summary of our detections and follow-up studies of three $\gamma$-ray-emitting NLS1s: SDSS J211852.96-073227.5 with flaring $\gamma$-ray radiation [U, [1] and SDSS J122222.55+041315.7 with the highest redshift by far $(\mathrm{z} \sim 1)[3]$, along with the prototype $1 \mathrm{H} 0323+342$ [四, []]. And we will discuss their multiwavelength properties and variability properties, including implications from high-energy observations in $\gamma$-rays and X-rays, infrared and radio properties, and correlated variability between several wavebands.
\end{abstract}

Revisiting narrow-line Seyfert 1 galaxies and their place in the Universe - NLS1 Padova

9-13 April 2018

Padova Botanical Garden, Italy

\footnotetext{
* Speaker.
} 


\section{Introduction}

It has been ten years since the Fermi discovery of narrow-line Seyfert 1 (NLS1) galaxies as a new class of $\gamma$-ray-emitting active galactic nuclei (AGNs) [6]. Distinctly different from the paradigm that powerful relativistic jets are generally originated from typical radio-loud (RL) AGNs like blazars and radio galaxies, NLS1s are a class of AGNs with black hole (BH) masses 1-2 orders of magnitude smaller and Eddington ratios near or above Eddington limits inferred from their smaller widths of the broad Balmer lines.

The extreme properties of NLS1s in AGN parameter space sparked great attention from the community and systematic searches have been conducted to look for RL NLS1s with possible

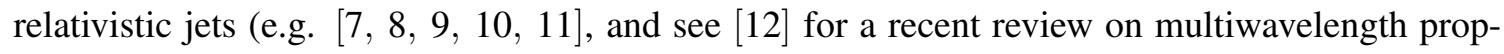
erties of RL NLS1s). RL NLS1s are rare (with $\sim 7 \%$ in fraction) compared to the RL subset of normal broad-line AGNs (15-20\%) [ []]. The further study of some very RL NLS1s (with radio loudness $\mathrm{R}>100$ ) has revealed their blazar-like properties, indicating the presence of relativistic jets (e.g. [G, 四, [3]). With the help of the Fermi $\gamma$-ray space telescope (hereinafter Fermi) and optical spectroscopic surveys like the Sloan Digital Sky Survey (SDSS), more than a dozen of $\gamma$-ray-emitting NLS1s have been detected which confirmed the existence of relativistic jets in this new class of $\gamma$-ray loud AGNs. In this paper, I will give a summary of three $\gamma$-ray-emitting NLS1s found and studied by our group in recent years, including SDSS J211852.96-073227.5 (hereinafter J2118-0732, a $\gamma$-ray flaring NLS1 galaxy [प] which was also independently discovered by [z]), SDSS J122222.55+041315.7 (hereinafter J1222+0413, by far the most distant $\gamma$-rayemitting NLS1 galaxy at redshfit of $z=0.966$ [3] ), and 1H 0323 +342 (a prototype of NLS1-blazar

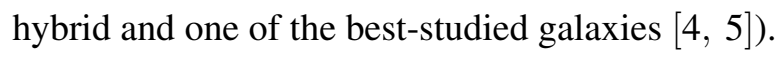

\section{2. $\gamma$-ray variability}

So far, only a small number of $\gamma$-ray loud NLS1s have been discovered. The comprehensive $\gamma$-ray study has been applied to several sources with the strongest $\gamma$-ray emission.

Our analysis from [U] has shown that $\mathbf{J} 2118-0732$ was in a relatively high-flux state during 2009-2013 and dimmed below the detection limit of the Large Area Telescope (LAT) onboard the Fermi after 2013. During the active 4 years, J2118-0732 was detected sporadically by the Fermi-LAT, with a flaring event emerging within $20 \mathrm{~d}$ and quickly dropped to a lower state (see the left panel in Fig. W). Rapid flares on time-scale of days have also been detected in $1 \mathrm{H} 0323+342$ [प4]. Since $\gamma$-ray flares and long-term variability are common in blazars, more $\gamma$-loud NLS1s will be detected as the Fermi continues to measure.

\section{X-ray spectra and variability}

RL NLS1s have systematically harder X-ray spectra (with the average photon index of $\Gamma_{\mathrm{X}}=$ $2.0 \pm 0.5)$ compared with radio-quiet NLS1s $\left(\Gamma_{X}=2.7\right)$ [प5], hinting perhaps at a jet origin. For example, the X-ray photon indices are $\Gamma_{\mathrm{X}}=1.1-1.5$ for $\mathrm{J} 1222+0413$ [3] and $\Gamma_{\mathrm{X}} \sim 1.7$ for J2118-0732 [U]. 1H 0323+342 has a softer X-ray spectrum $\left(\Gamma_{X} \sim 1.9\right)$ than other $\gamma$-ray detected NLS1s, indicating that the disk/corona may make a significant contribution in the X-ray band [5]. 


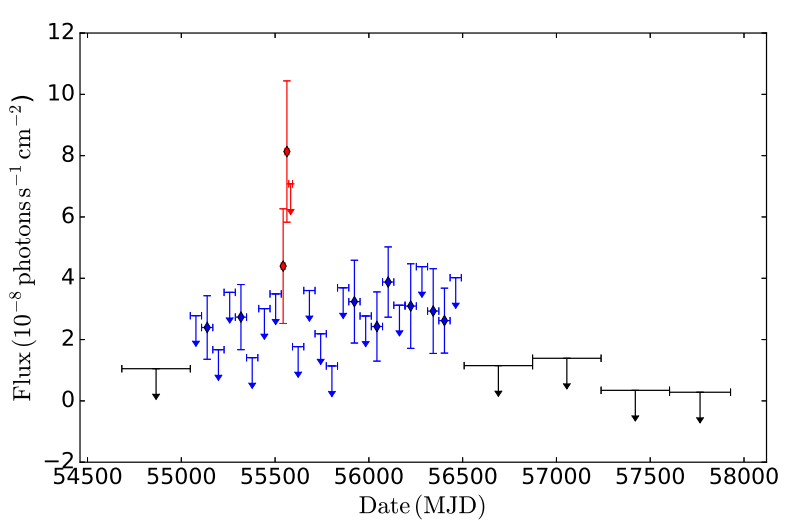

(a)

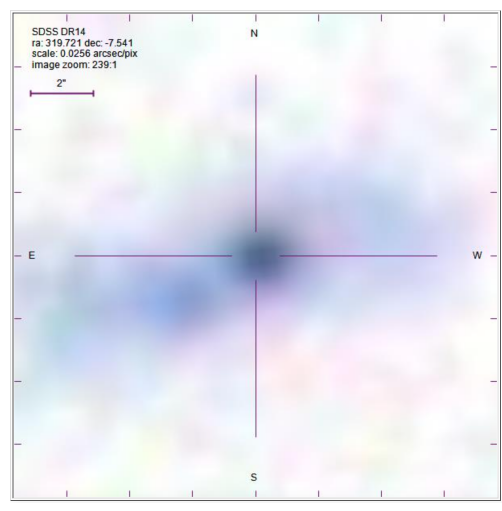

(b)

Figure 1: $\gamma$-ray light curve (left) and SDSS image (right) of J2118-0732. In the left panel, $\gamma$-ray fluxes were obtained in the $0.1-300 \mathrm{GeV}$ band by the Fermi-LAT with 1-yr bins in black, 60-d bins in blue, and $20-\mathrm{d}$ bins in red from fig. 1 in $[\mathbf{U}$. Diamonds refer to detections with $1 \sigma$ errors and arrows refer to $95 \%$ upper limits. In the right panel, optical image of J2118-0732 were obtained from SDSS Data Release 14 database.

A soft X-ray excess has been detected in most $\gamma$-loud NLS1s that have good-quality X-ray spectra, with exceptions for J2118-0732 [U] and PKS 2004-447 [16]. Given the prevalence of soft excesses in radio-quiet NLS1s, they are likely also common in RL NLS1s. However, not all NLS1s have steep soft X-ray spectra, and in RL NLS1s, intrinsic soft excesses may sometimes escape detections, when the jet emission is in a high-state.

Significant X-ray variability has also been detected in $\gamma$-ray loud NLS1s at multiple timescales. The X-ray flux integrated from 0.3 to $10 \mathrm{keV}$ of $\mathrm{J} 2118-0732$ dropped by a factor of $\sim 3$ between the two XMM-Newton observations separated by about five months [U]. A significant $\mathrm{X}$-ray variability on time-scales from ks to years has been detected in $1 \mathrm{H} 0323+342$ [5].

\section{Intraday infrared variability}

Both J2118-0732 and J1222+0413 are detected to have intraday infrared variability by studying the Wide-field Infrared Survey Explorer (WISE) data [U, []]. Such rapid infrared variability is also detected in some other RL NLS1s [U]]. And those sources with intraday infrared variability are usually the ones with the largest radio loudness and the highest radio brightness temperature. The detection of such a short variability time-scale can set an upper limit on the size of the infraredemitting region to $\leq 10^{-3} \mathrm{pc}$, which is significantly smaller than the scale of the torus but consistent with that of the jet-emitting size.

\section{Radio properties}

$1 \mathrm{H} 0323+342$ is a strong radio source with a flux density of $304-581 \mathrm{mJy}$ at $5 \mathrm{GHz}$, and it has a flat radio spectrum up to at least $10 \mathrm{GHz}\left[\alpha_{\text {rad }} \approx 0.1\left(S_{v} \propto v^{\alpha}\right)\right]$ and significant flux variations [ [ 9 ]. The polarization levels of $1 \mathrm{H} 0323+342$ are ranging from $3 \%$ to $5 \%$ at multiple radio bands. The 
radio spectrum of $\mathrm{J} 1222+0413$ is also very flat $\left(\alpha_{\mathrm{rad}} \approx 0.3\right)$ with the core flux density of $600 \mathrm{mJy}$ at $1.4 \mathrm{GHz}$ and variations on time-scales from years to decade at $\mathrm{GHz}$ bands [3] . As for J2118-0732, the $1.4 \mathrm{GHz}$ flux density is $96 \mathrm{mJy}$ and the polarized flux density is $3.2 \mathrm{mJy}$, corresponding to a fractional polarization of $\sim 3 \%$. The source shows a steep spectrum at lower frequencies, however, a flat/inverted spectrum above $1.4 \mathrm{GHz}$ [प]. The common feature of the flat radio spectrum at higher frequencies among RL NLS1s may also indicate a jet nature, where the flat spectrum could be the result of a superposition of several jet components [ए8].

These three sources have all been observed by the Very Long Baseline Array (VLBA). The high-resolution images of $1 \mathrm{H} 0323+342$ and $\mathrm{J} 1222+0413$ reveal a core-jet structure [ 9 , प9] while that of J2118-0732 shows an unresolved core only ${ }^{1}$, supporting the core nature. This is consistent with the parsec-scale radio properties of a sample of RL NLS1s studied from VLBA observations [20].

\section{Correlated multiwavelength variability}

By studying the light curves at multiple wavelengths, coordinated variation patterns are found from some $\gamma$-ray NLS1s, indicating the connections among accretion discs, coronas, and jets. With the help of intensive Swift monitoring observations, a statistically significant correlation between X-ray and ultraviolet (UV) $w 2$ bands was found for $1 \mathrm{H} 0323+342$ [5]. And a further cross-correlation method resulted in a possible time lag $\tau=0.6_{-1.0}^{+2.7}$ day with the X-rays tentatively leading the UV. However, the uncertainty of the time lag is quiet large and the lag is statistically consistent with zero. For another interesting source J2118-0732, two XMM-Newton observations separated by about five months were proposed and carried out [U]. Together with the WISE data, two broadband simultaneous spectral energy distributions (SEDs) were constructed and showed the synchronous drop from infrared to X-rays in five months.

\section{Host galaxy}

Optical observations of the host galaxies of most RL NLS1s have not yet been carried out, so we know very little about their hosts. Only a few $\gamma$-NLS1s have been imaged recently. For example, $1 \mathrm{H} 0323+342$ shows a one-armed spiral galaxy [ [4] or possibly resides in a system disturbed by merging [2], [2]]. The SDSS imaging of J2118-0732 shows an extended galaxy structure with a disturbed morphology, possibly suggesting a recent merger (see the right panel in Fig. W). This may suggest that mergers could be important for the production of relativistic jets in RL NLS1s.

\section{Broadband SED}

The broadband SEDs of $\gamma$-ray NLS1s are representative of those blazars which exhibit double distinct peaks. And they are usually explained by the synchrotron radiation and the inverse Compton scattering of the relativistic electrons in the jets. Apart from the powerful jet emission, the disc/corona emission is also very strong in some RL NLS1s, contributing to the optical/UV and

\footnotetext{
${ }^{1}$ http://astrogeo.org/cgi-bin/imdb_get_source.csh?source=J2118-0732
} 
$\mathrm{X}$-ray fluxes. The simple one-zone leptonic jet model is often used to account for the broadband emission from GeV-NLS1 galaxies, sometimes accompanied by disc/corona emission to explain the big blue bump and softer X-ray spectrum.

The broadband SED of 1H 0323+342 has been modelled in several works (e.g. [1], [44, [23], [24]), which could be well explained by the jet plus disc/corona model (see the right panel in Fig. $\mathbb{Z}$ ). And the broadband SEDs of J2118-0732 (see the left panel in Fig. W) and J1222+0413 can be well fitted with the jet+disc model [ [U, [3]. However, the question still remains whether the dissipation region of the jet locates near the $\mathrm{BH}$ or farther away in the broad line region or torus.

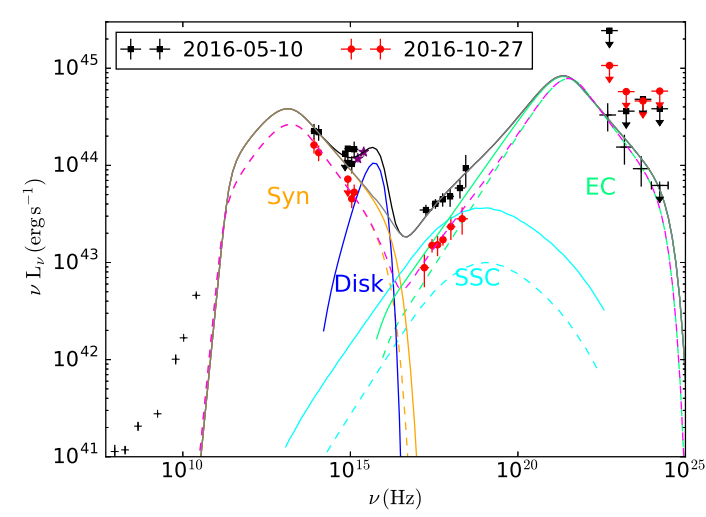

(a)

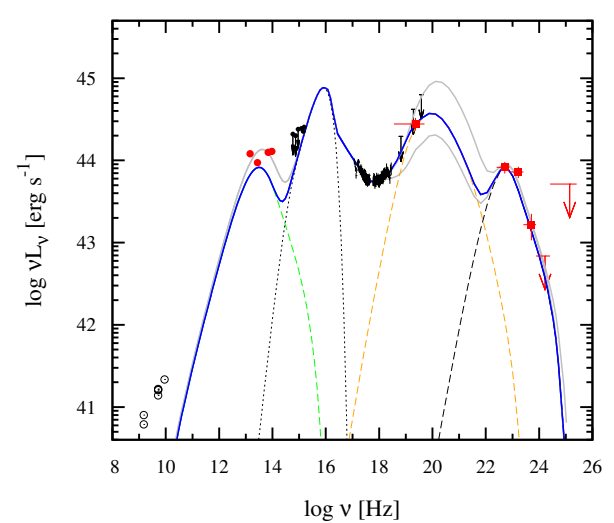

(b)

Figure 2: Broadband SEDs of J2118-0732 (left) and 1H 0323+342 (right) and their SED modellings. In the left panel, the black squares and red dots are measurements of J2118-0732 at different epochs. The black and magenta lines are their best-fitting models. In the right panel, data from optical/UV to X-rays are quasi-simultaneous while others are not. The blue solid line is the best-fitting model and the gray solid lines are the contributions from the SED modelling results after the hard X-rays were varied by a factor of 2 . The green, orange, and black dashed lines represent the synchrotron, synchrotron-self-Compton (SSC), and External Compton (EC) components, respectively. The black dotted line indicates the disc model.

\section{Summary}

In the last decade, we have seen a swarm of investigations of RL NLS1s, with the number of $\gamma$-ray-emitting NLS1s growing to $\sim 14$ or more detected by the Fermi-LAT telescope (see table 1 of [12]). Here, we have summarized our results of three $\gamma$-ray-emitting NLS1s. We have found blazar-like properties in these galaxies, including the long-term variability with sporadic flares in $\gamma$-rays, flat spectra at radio and X-ray bands, and rapid and correlated variability at several wavebands. Together with the NLS1 nature from optical spectroscopy, they constitute a very interesting class of objects called "NLS1-blazar hybrid". With their extreme distributions in AGN parameter space, RL NLS1s allow us to explore the crucial questions regarding the formation and evolution of relativistic jets under smaller BH masses and higher accretion rates than classical blazars as well as the coupling mechanism of jets and accretion flows. Further observations from the Fermi-LAT, optical and radio surveys would result in more RL NLS1s, particularly for those $\gamma$-ray loud NLS1s, and will shed more light on the jet properties by systematic studies at multiple wavelengths. 


\section{Acknowledgements}

This conference has been organized with the support of the Department of Physics and Astronomy "Galileo Galilei", the University of Padova, the National Institute of Astrophysics INAF, the Padova Planetarium, and the RadioNet consortium. RadioNet has received funding from the European Union's Horizon 2020 research and innovation programme under grant agreement No 730562. This conference proceeding has made use of the data from the Fermi, XMM-Newton, SDSS, WISE, and the Astrogeo VLBI FITS image database.

\section{References}

[1] H. Yang, W. Yuan, S. Yao, Y. Li, J. Zhang, H. Zhou et al., SDSS J211852.96-073227.5: a new $\gamma$-ray flaring narrow-line Seyfert 1 galaxy, MNRAS 477(2018) 5127 [15801.03963].

[2] V. S. Paliya, M. Ajello, S. Rakshit, A. K. Mandal, C. S. Stalin, A. Kaur et al., Gamma-Ray-emitting Narrow-line Seyfert 1 Galaxies in the Sloan Digital Sky Survey, ApJL853 (2018) L2 [11801.01905].

[3] S. Yao, W. Yuan, H. Zhou, S. Komossa, J. Zhang, E. Qiao et al., Identification of a new $\gamma$-ray-emitting narrow-line Seyfert 1 galaxy, at redshift $\sim 1$, MNRAS 454(2015)L16] [1509.03030].

[4] H. Zhou, T. Wang, W. Yuan, H. Shan, S. Komossa, H. Lu et al., A Narrow-Line Seyfert 1-Blazar Composite Nucleus in 2MASX J0324+3410, ApJL658(2007) L13] [astro-ph/0702329].

[5] S. Yao, W. Yuan, S. Komossa, D. Grupe, L. Fuhrmann and B. Liu, The $\gamma$-Ray Detected Narrow-line Seyfert 1 Galaxy 1H 0323+342: Swift Monitoring and Suzaku Spectroscopy, AJ 150(2015) 23 [1505.01269].

[6] A. A. Abdo, M. Ackermann, M. Ajello, M. Axelsson, L. Baldini, J. Ballet et al., Fermi/Large Area Telescope Discovery of Gamma-Ray Emission from a Relativistic Jet in the Narrow-Line Quasar $P M N$ J0948+0022, ApJ 699 (2009)976 [0905 . 4558].

[7] S. Komossa, W. Voges, D. Xu, S. Mathur, H.-M. Adorf, G. Lemson et al., Radio-loud Narrow-Line Type 1 Quasars, AJ132(2006)531 astro-ph/0603680].

[8] H. Zhou, T. Wang, W. Yuan, H. Lu, X. Dong, J. Wang et al., A Comprehensive Study of 2000 Narrow Line Seyfert 1 Galaxies from the Sloan Digital Sky Survey. I. The Sample, $4 p J S 166(2006) 128$ [astro-ph/0603759].

[9] W. Yuan, H. Y. Zhou, S. Komossa, X. B. Dong, T. G. Wang, H. L. Lu et al., A Population of Radio-Loud Narrow-Line Seyfert 1 Galaxies with Blazar-Like Properties?, ApJ 685 (2008) 801 [0806.3755].

[10] S. Rakshit, C. S. Stalin, H. Chand and X.-G. Zhang, A Catalog of Narrow Line Seyfert 1 Galaxies from the Sloan Digital Sky Survey Data Release 12, ApJS 229 (2017) 39 [15704.07700].

[11] S. Chen, M. Berton, G. La Mura, E. Congiu, V. Cracco, L. Foschini et al., Probing narrow-line Seyfert 1 galaxies in the southern hemisphere, A\&A615 (2018) A167] [1801.07234]].

[12] S. Komossa, Multi-Wavelength Properties of Radio-loud Narrow-Line Seyfert 1 Galaxies, arXiv e-prints (2018) [10807.03666].

[13] H.-Y. Zhou, T.-G. Wang, X.-B. Dong, Y.-Y. Zhou and C. Li, SDSS J094857.3+002225: A Very Radio Loud, Narrow-Line Quasar with Relativistic Jets?, ApJ584 (2003) 147. 
[14] V. S. Paliya, S. Sahayanathan, M. L. Parker, A. C. Fabian, C. S. Stalin, A. Anjum et al., The Peculiar Radio-loud Narrow Line Seyfert 1 Galaxy 1H 0323+342, ApJ789(2014) 143 [1405.0715].

[15] L. Foschini, M. Berton, A. Caccianiga, S. Ciroi, V. Cracco, B. M. Peterson et al., Properties of flat-spectrum radio-loud narrow-line Seyfert 1 galaxies, A\&A 575(2015) A13 [11409 . 3716].

[16] A. Kreikenbohm, R. Schulz, M. Kadler, J. Wilms, A. Markowitz, C. S. Chang et al., The gamma-ray emitting radio-loud narrow-line Seyfert 1 galaxy PKS 2004-447. I. The X-ray View, A\&A 585 (2016) A9] [1509.03735].

[17] N. Jiang, H.-Y. Zhou, L. C. Ho, W. Yuan, T.-G. Wang, X.-B. Dong et al., Rapid Infrared Variability of Three Radio-loud Narrow-line Seyfert 1 Galaxies: A View from the Wide-field Infrared Survey Explorer, ApJL 759(2012)L31] [1210.2800].

[18] A. Konigl, Relativistic jets as X-ray and gamma-ray sources, ApJ 243 (1981) 700.

[19] M. L. Lister, Radio Properties of Narrow-Line Seyfert 1 Galaxies, arXiv e-prints (2018) [1805.05258].

[20] M. Gu, Y. Chen, S. Komossa, W. Yuan, Z. Shen, K. Wajima et al., The Radio Properties of Radio-loud Narrow-line Seyfert 1 Galaxies on Parsec Scales, ApJS 221 (2015) 3 [प509.01889].

[21] S. Antón, I. W. A. Browne and M. J. Marchã, The colour of the narrow line Sy1-blazar 0324+3410, A\&A 490 (2008) 583 [0907. 2400$]$ ].

[22] J. León Tavares, J. Kotilainen, V. Chavushyan, C. Añorve, I. Puerari, I. Cruz-González et al., The Host Galaxy of the Gamma-Ray Narrow-line Seyfert 1 Galaxy $1 H$ 0323+342, ApJ795(2014) 58 [11409.2518].

[23] A. A. Abdo, M. Ackermann, M. Ajello, L. Baldini, J. Ballet, G. Barbiellini et al., Radio-Loud Narrow-Line Seyfert 1 as a New Class of Gamma-Ray Active Galactic Nuclei, ApJL707(2009) L142 [0911.3485].

[24] D. Kynoch, H. Landt, M. J. Ward, C. Done, E. Gardner, C. Boisson et al., The relativistic jet of the $\gamma$-ray emitting narrow-line Seyfert 1 galaxy $1 H$ 0323+342, MNRAS 475 (2018) 404 [1]712.01799]. 